PROCEEDINGS OF THE

AMERICAN MATHEMATICAL SOCIETY

Volume 128, Number 10, Pages 3115-3124

S 0002-9939(00)05346-6

Article electronically published on March 2, 2000

\title{
BAIRE AND VOLTERRA SPACES
}

\author{
GARY GRUENHAGE AND DAVID LUTZER
}

(Communicated by Alan Dow)

\begin{abstract}
In this paper we describe broad classes of spaces for which the Baire space property is equivalent to the assertion that any two dense $G_{\delta^{-}}$ sets have dense intersection. We also provide examples of spaces where the equivalence does not hold. Finally, our techniques provide an easy proof of a new internal characterization of perfectly meager subspaces of $[0,1]$ and characterize metric spaces that are always of first category.
\end{abstract}

\section{INTRODUCTION}

Recall that a topological space $X$ is a Baire space if the intersection of any sequence of dense open subsets of $X$ is dense. It follows immediately that the intersection of countably many dense $G_{\delta}$-subsets of a Baire space $X$ must be dense in $X$. A weaker condition is the that intersection of any two dense $G_{\delta}$-sets of $X$ must be dense in $X$, and that is the definition of a Volterra space GP, GGP. Obviously, any Baire space is Volterra, and in this paper we study when the converse holds.

The term "Volterra space" was first used in GP. That name was chosen in the light of an 1881 paper by V. Volterra $[\mathrm{V}]$ who proved that if $f: R \rightarrow R$ is any function such that both $C(f)=\{x \in R: f$ is continuous at $x\}$ and $D(f)=R-C(f)$ are dense in $R$, then there cannot be a function $g: R \rightarrow R$ such that $C(g)=D(f)$ and $D(g)=C(f)$. The key idea in Volterra's proof was that the intersection of two dense $G_{\delta}$ subsets of $R$ must be dense in $R$.

In Section 2 we give properties of a space $X$ which guarantee that $X$ is a Baire space if and only if $X$ is Volterra. In Corollary 2.8, we apply our conditions to answer a question of Piotrowski concerning metric spaces. We show that the equality "Baire = Volterra" holds (i) for any space that has a dense metrizable subspace, (ii) for any Lasnev space, and (iii) for any metacompact Moore space. In Section 3 we give three examples of spaces that are Volterra but not Baire. The first is countable and regular; the second is a Lindelöf, hereditarily paracompact, linearly ordered topological space; and the third is first countable and paracompact. We

Received by the editors May 18, 1998 and, in revised form, November 24, 1998.

2000 Mathematics Subject Classification. Primary 54E52; Secondary 54E20, 54E25, 54E30, 54E35, 54H05, 54F65.

Key words and phrases. Baire space, Volterra space, metric space, Moore space, Lasnev space, linearly ordered topological space, perfectly meager set, $\lambda$-set, always first category.

Research of the first author partially supported by NSF grant DMS-9704849, Auburn University. 
do not know whether the equality "Baire = Volterra" holds for arbitrary Moore spaces.

The final section of our paper uses a lemma from Section 2 to provide a short proof of a new internal characterization of perfectly meager subspaces of $[0,1]$ that was originally obtained as a corollary of more technical constructions in $[\mathrm{BHL}$, and to characterize metric spaces that are always of first category. Relevant definitions appear in Section 4.

All spaces in our paper are assumed to be regular and $T_{1}$, unless otherwise noted. Our terminology and notation follow E] and G1.

\section{Spaces In Which "Volterra = Baire"}

Clearly, any Baire space is Volterra. The goal of this section is to give a large class of spaces in which the converse holds. Recall from [H] that a space $X$ is resolvable if $X$ contains two disjoint dense subsets. The next result is due to Pytkeev $[\mathrm{P}]$.

2.1 Lemma. Any dense-in-itself subspace of a sequential space is resolvable.

2.2 Lemma. Let $M$ be a dense subset of a regular $T_{1}$-space $Y$, such that every point of $M$ is $G_{\delta}$ in $Y$. Suppose $\{p\} \cup M$ is homeomorphic to a subspace of a Hausdorff sequential space $S$ (we are not assuming $p \in Y$ ), and $p \in \operatorname{cl}_{S}(M)$. Then there is a countable subset $C(p, M)$ of $M$ with $p \in c l_{S}(C(p, m))$ and such that $C(p, M)$ is $G_{\delta}$ in $Y$.

Proof. For a subset $A$ of $S$, let $\operatorname{seq}(A)$ be the set of all limits in the space $S$ of convergent sequences $\left\{a_{n}: n \in \omega\right\} \subset A$. Then define $A^{0}=A, A^{\alpha+1}=\operatorname{seq}\left(A^{\alpha}\right)$, and $A^{\beta}=\bigcup\left\{A^{\alpha}: \alpha<\beta\right\}$ if $\beta$ is a limit ordinal. Recall that $S$ sequential means that $c_{S}(A)=\bigcup\left\{A^{\alpha}: \alpha<\omega_{1}\right\}$.

Let $\alpha$ be least such that $p \in M^{\alpha}$. Call $\alpha$ the "sequential order of $p$ w.r.t. $M$ ", and note that $\alpha$ is a successor. If $\alpha=1$, then there is a sequence $J=\left\{m_{k}: k \in \omega\right\}$ of points in $M$ converging to $p$. Since $J$ is relatively discrete in $M$, it follows from the regularity of $Y$ that there is a disjoint collection $\left\{U_{k}: k \in \omega\right\}$ of open subsets of $Y$ with $m_{k} \in U_{k}$ for every $k$. Since each point of $M$ is $G_{\delta}$ in $Y$, it follows that $J$ is $G_{\delta}$ in $Y$. Thus we can take $C(p, M)=J$.

Suppose $\alpha>1$ and the lemma holds whenever the sequential order of $p$ in $M$ is less than $\alpha$. Let $\alpha=\gamma+1$. Then there is a sequence $\left\{m_{k}: k \in \omega\right\} \subset M^{\gamma}$ converging to $p$ in $S$. Since $S$ is Hausdorff, we may assume that there are disjoint open (in $S$ ) sets $V_{k}, k \in \omega$, with $m_{k} \in V_{k}$. Note that the sequential order of $m_{k}$ is the same w.r.t. $V_{k} \cap M$ as w.r.t. $M$. For each $k$ let $V_{k}^{*}$ be open in $Y$ such that $V_{k}^{*} \cap M=V_{k} \cap M$, and note that the $V_{k}^{*}$ 's are also disjoint (since $M$ is dense in $Y)$. Now apply the induction hypothesis with $p=m_{k}, Y=V_{k}^{*}$, and $M=M \cap V_{k}$ to obtain a countable $C\left(m_{k}, M \cap V_{k}\right)$ contained in $M \cap V_{k}$ which is $G_{\delta}$ in $V_{k}^{*}$ (and hence in $Y)$. Let $C(p, M)=\bigcup_{k \in \omega} C\left(m_{k}, M \cap V_{k}\right)$. Clearly $p \in c l_{S}(C(p, M))$, and since the $V_{k}^{*}$ 's are disjoint open sets in $Y$, it follows that $C(p, M)$ is $G_{\delta}$ in $Y$.

2.3 Lemma. Suppose $\mathcal{U}$ is a point-finite collection of open subsets of a space $X$ and that for each $U \in \mathcal{U}$ we have a $G_{\delta}$-subset $G(U) \subset U$. Then $S=\bigcup\{G(U): U \in \mathcal{U}\}$ is a $G_{\delta}$-subset of $X$.

Proof. For each $U \in \mathcal{U}$, write $G(U)=\bigcap\{G(n, U): n \geq 1\}$ where each $G(n, U)$ is an open subset of $U$ with $G(n+1, U) \subset G(n, U)$. Define $H_{n}=\bigcup\{G(n, U): U \in \mathcal{U}\}$. Then point-finiteness of $\mathcal{U}$ yields that $\bigcap\left\{H_{n}: n \geq 1\right\}=S$. 
2.4 Lemma. Suppose $X$ is regular, points are $G_{\delta}$, and $X$ has a dense subspace $D=\bigcup\left\{D_{n}: n \geq 1\right\}$ satisfying:

(a) $D$ is homeomorphic to a subspace of a Hausdorff sequential space;

(b) for each $n \geq 1$ there is a collection $\left\{V(d, n): d \in D_{n}\right\}$ of open subsets of $X$ that is point-finite in $X$ and has $\{d\}=V(d, n) \cap D_{n}$.

If $X$ is of the first category in itself, then $D$ contains a subspace $E$ that is dense in $X$ and is a $G_{\delta}$-subset of $X$.

Proof. Because $X$ is a first category space, there is a sequence $\left\{G_{n}: n \geq 1\right\}$ of dense open subsets of $X$ such that $\bigcap\left\{G_{n}: n \geq 1\right\}=\emptyset$. We may assume that $G_{n+1} \subset G_{n}$. Then $X$ has no isolated points so that neither does the dense subspace $D$.

We will show that $D$ contains a dense, $G_{\delta}$ subset $E$ of $X$. Let $\mathcal{V}_{n}=\{V(n, d)$ : $\left.d \in D_{n}\right\}$ be the point-finite collection given by (b). Because $G_{n} \cap D$ is dense in $X$, for each $d \in D_{n}$ the conditions of Lemma 2.2 are satisfied with $Y=G_{n} \cap(V(d, n))$, $M=D \cap G_{n} \cap(V(d, n)-\{d\}), S$ any Hausdorff sequential space containing $D$, and $p=d$. Thus there is a countable subset $K(d, n)$ of $G_{n} \cap D \cap(V(d, n)-\{d\})$ which is $G_{\delta}$ in $Y$ and so also in $X$, and $d \in \operatorname{cl}_{S}(K(d, n))$. Since $\{d\} \cup K(d, n) \subset D$ and $D$ has the same topology in $X$ as in $S$, we also have $d \in \operatorname{cl}_{X}(K(d, n))$. Now by (2.3), each set $H_{n}=\bigcup\left\{K(d, n): d \in D_{n}\right\}$ is a $G_{\delta}$-subset of $X$. Because the collection $\left\{G_{n}: n \geq 1\right\}$ is point-finite in $X$, it follows again from (2.3) that the set $E=\bigcup\left\{H_{n}: n \geq 1\right\}$ is a $G_{\delta}$-subset of $X$. Observe that $E \subset D$ and that each point of each set $D_{n}$ is a limit point of $E$, showing that $E$ is dense in $X$, as required.

2.5 Corollary. If $X$ is a metric space that is first category in itself, and if $Y$ is a dense subset of $X$, then $Y$ contains a dense, $G_{\delta}$-subset of $X$ that is $\sigma$-closed discrete in $X$.

Proof. The dense subspace $Y$ contains a dense subset $D$ that is $\sigma$-closed discrete in $X$. Now apply (2.4) to the subspace $D$.

2.6 Proposition. Suppose $X$ is regular and has a dense subspace $D=\bigcup\left\{D_{n}\right.$ : $n \geq 1\}$ satisfying:

(a) $D$ is homeomorphic to a subspace of a Hausdorff sequential space;

(b) for each $n \geq 1$ there is a collection $\left\{V(d, n): d \in D_{n}\right\}$ of open subsets of $X$ that is point-finite in $X$ and has $V(d, n) \cap D_{n}=\{d\}$ for each $d \in D_{n}$.

Then $X$ is a Baire space if and only if $X$ is Volterra.

Proof. Any Baire space is Volterra, so it is enough to prove the converse. Suppose $X$ is Volterra and yet there is a sequence $\left\{G_{n}: n \geq 1\right\}$ of dense open subsets of $X$ such that $G_{n+1} \subset G_{n}$ and $\bigcap\left\{G_{n}: n \geq 1\right\}$ is not dense. Then there is a non-empty open subset $Y \subset X$ such that $Y \cap \bigcap\left\{G_{n}: n \geq 1\right\}=\emptyset$. Observe that the set $D \cap Y$ is dense in $Y$ and satisfies both (a) and (b) above. Replacing $X$ by its subspace $Y$ if necessary, we may assume that $\bigcap\left\{G_{n}: n \geq 1\right\}=\emptyset$. It follows that $X$ has no isolated points. Hence neither does the dense subspace $D$.

Apply (2.1) to $D$ to find two disjoint, dense subspaces $D_{1}, D_{2}$ of $D$. Apply (2.4) to each $D_{i}$ to find a subspace $E_{i} \subset D_{i}$ that is dense in $X$ and is a $G_{\delta}$-subset of $X$. But then we have two disjoint dense $G_{\delta}$-subsets of $X$, and that is impossible because $X$ is Volterra.

2.7 Remark. Condition (b) in (2.6) can be weakened to "For each $n \geq 1$ there is a collection $\left\{V(d, n): d \in D_{n}\right\}$ of open subsets of $X$ with $d \in V(d, n) \cap D_{n}$ and such that for each $x \in X$, the set $\left\{d \in D_{n}: x \in V(d, n)\right\}$ is finite." 
Our next result gives several broad classes $\mathcal{S}$ of spaces such that any $X \in \mathcal{S}$ is a Baire space if and only if $X$ is Volterra. In particular, (2.8) gives an affirmative answer to a question posed by Gauld, Greenwood, and Piotrowski [GGP] who asked whether a Volterra metric space must be a Baire space.

2.8 Corollary. A Volterra space $X$ is Baire if $X$ belongs to any one of the following classes:

(a) $X$ has a dense subspace $Y$ that is a strongly collectionwise Hausdorff, sequential, and has a relatively $\sigma$-closed discrete dense subset;

(b) $X$ has a dense metrizable subspace;

(c) $X$ is a Lasnev space, i.e., a closed continuous image of a metric space;

(d) $X$ is a metacompact sequential space that has a $\sigma$-closed-discrete dense set;

(e) $X$ is a metacompact Moore space or, more generally, a metacompact semistratifiable sequential space;

(f) $X$ is separable and sequential.

Proof. Case (a) follows from the fact that if $Y$ is as described, then $Y$ has a dense subset $D=\bigcup\left\{D_{n}: n \geq 1\right\}$ such that each $D_{n}$ is a relatively closed, discrete subspace of $Y$. Because $Y$ is strongly collectionwise Hausdorff, for each $n$ we may find a collection $\left\{W(d, n): d \in D_{n}\right\}$ of pairwise disjoint, relatively open subsets of $Y$, with $W(d, n) \cap D_{n}=\{d\}$ for each $d \in D_{n}$. Write $W(d, n)=Y \cap V(d, n)$ where $V(d, n)$ is an open subset of $X$. Because $Y$ is dense in $X$, the collection $\left\{V(d, n): d \in D_{n}\right\}$ must be pairwise disjoint and hence point-finite in $X$. Now (2.6) applies to complete the proof of (a). Given (a), assertion (b) is immediate and (c) follows from (a) by letting $Y=X$.

Assertion (d) is an immediate consequence of (2.6) because metacompactness of $X$ allows us to expand each level of the $\sigma$-closed discrete dense set $D=\bigcup\left\{D_{n}: n \geq\right.$ 1 ) to a point-finite open collection in $X$, as required in (2.6). To prove assertion (e), recall that any semi-stratifiable space has a dense $\sigma$-closed discrete subspace so that (d) yields the desired conclusion. Finally, assertion (f) follows from (a) by letting $Y$ be any countable dense subset of $X$.

2.9 Remark. Arhangel'skii and Nedev [AN] noted that if $V=L$, then every normal semi-metric space contains a dense metrizable subspace. Hence, $V=L$ yields that if $X$ is a normal semi-metric space, then $X$ is a Baire space if and only if $X$ is Volterra.

2.10 Remark. Example 3.1 provides a countable, regular space that is Volterra but not Baire, showing that the sequentiality conditions in 2.6 and 2.8 cannot be eliminated entirely.

2.11 Questions. (a) Is it true that any Volterra Moore space must be a Baire space?

(b) Is it true that a space $X$ must be a Baire space provided $X$ is Volterra and has a dense subspace that is developable and metacompact? (If the dense subspace is developable and screenable, then the answer is affirmative [He.)

(c) Must $X$ be Baire if $X$ is stratifiable and Volterra?

(d) Suppose $X$ is a $\sigma$-space and first category in itself. Must every dense subset $D$ of $X$ contain a dense subset $E$ which is $G_{\delta}$ in $X$ ? (By 2.4, the answer is positive if $X$ is metacompact and sequential.)

An earlier draft of this paper pointed out that if a Moore space has a dense subspace $D$ as in (2.6), then $D$ is itself a metacompact Moore space, and asked 
whether every Moore space has a dense, metacompact subspace. G.M. Reed and D. McIntyre have provided a counterexample in $\mathrm{RM}$.

\section{EXAMPLES}

3.1 Example. There is a countable regular space that is Volterra but not Baire.

Proof. In vD, van Douwen constructed a space $X$ with the following properties:

(a) $X$ is countable, regular, and $\mathrm{T}_{1}$;

(b) every non-empty open subset of $X$ is infinite;

(c) if $A, B \subset X$ have no isolated points in their relative topologies, and if $A \cap B=$ $\emptyset$, then no point of $X$ is a limit point of both $A$ and $B$ so that $\operatorname{cl}(A) \cap \operatorname{cl}(B)=\emptyset$. (Van Douwen called such a space "ultra-disconnected." Note that this property implies that $X$ contains no convergent sequences.)

Being a countable space with no isolated points, $X$ is first category in itself. However, $X$ is Volterra. Indeed, even more is true, for suppose that $S$ and $T$ are any two dense subsets of $X$. If $S \cap T$ is not dense in $X$, then for some open $U \subset X$ we have $S \cap T \cap U=\emptyset$. Let $A=S \cap U$ and $B=T \cap U$. Because $A \cap B=\emptyset$, property (c) yields $\emptyset=\operatorname{cl}(A) \cap \operatorname{cl}(B) \supseteq U$ which is impossible.

Note that while this $X$ shows that Volterra and Baire are not equivalent in the class of $\sigma$-spaces, it does not answer question 2.11(d), since every subset of $X$ is $G_{\delta}$.

3.2 Example. There is a Lindelöf, hereditarily paracompact linearly ordered topological space that is Volterra but not Baire.

Proof. This space is a slight modification of [G2, Ex. 1.1]. Let $Z$ be the set of integers and let $X=\left\{f: \omega_{1} \rightarrow Z:\left\{\alpha<\omega_{1}: f(\alpha) \neq 0\right\}\right.$ is finite $\}$. Endow $X$ with the lexicographic order and the open interval topology of that ordering. For $f \in X$ and $\alpha<\omega_{1}$, let $B(f, \alpha)=\{g \in X: g(\beta)=f(\beta)$ for each $\beta \leq \alpha\}$. One can check that $\left\{B(f, \alpha): \alpha<\omega_{1}\right\}$ is a neighborhood base at $f$ for any $f \in X$. It follows that every $G_{\delta}$-subset of $X$ is open and therefore that $X$ is Volterra. Further, as in G2, $X$ is Lindelöf and hereditarily paracompact. However, $X$ is not a Baire space because $X=\bigcup\left\{X_{n}: n \geq 1\right\}$ where $X_{n}=\left\{f \in X:\left|\left\{\alpha<\omega_{1}: f(\alpha) \neq 0\right\}\right| \leq n\right\}$, and each $X_{n}$ is closed and nowhere dense.

3.3 Example. There is a first countable, completely regular, paracompact space that is a Volterra space and is not a Baire space.

Proof. The underlying set of this example will be $X=Q \times \mathcal{B}$ where $Q$ is the usual space of rational numbers and $\mathcal{B}$ is a certain branch space, described below. The topology $\tau$ for $X$ will be constructed inductively, starting with the usual product topology $\tau_{0}$ of $X$. Below we present a series of claims that verify properties of $(X, \tau)$. Claim 3 shows that $(X, \tau)$ is first countable; Claim 4 shows that the space is completely regular and $\mathrm{T}_{1}$, and Claim 5 shows that it is paracompact; Claim 6 shows that $(X, \tau)$ is not Baire; and Claim 7 shows that the space is Volterra.

Let $B$ be a bistationary subset of $\left[0, \omega_{1}\right)$ (i.e., both $B$ and $\left[0, \omega_{1}\right)-B$ are stationary) and let $\mathcal{T}=\left\{T \subset B: T\right.$ is a closed subset of $\left.\left[0, \omega_{1}\right)\right\}$. Each member of $\mathcal{T}$ is countable and contains its supremum. Further, $|\mathcal{T}|=2^{\omega}=\underline{c}$. Partially order $\mathcal{T}$ by "end extension", i.e. $S \prec T$ if and only if $[0, \sup (S)] \cap T=S$. For each $T \in \mathcal{T}$, 
let $V_{T}=\{S \in \mathcal{T}: T \prec S\}$ and let $\Phi$ be the topology on $\mathcal{T}$ for which the collection $\left\{V_{T}: T \in \mathcal{T}\right\}$ is a base. This auxiliary space is not even $\mathrm{T}_{1}$, but it does have one crucial property that we will need later, namely that the intersection of countably many dense open subsets of $(\mathcal{T}, \Phi)$ is a dense, open set. One proof appears in $[\mathrm{T}$. Lemma 9.12]; direct proofs are also possible, using the fact that $B$ is stationary.

By a "branch" of $\mathcal{T}$ we mean a maximal, linearly ordered (by $\prec$ ) subset of $\mathcal{T}$. Because $\left[0, \omega_{1}\right)-B$ is stationary, each branch is countable. Let $\mathcal{B}=\{b: b$ is a branch of $\mathcal{T}\}$ and define $[T]=\{b \in \mathcal{B}: T \in b\}$ for each $T \in \mathcal{T}$. Topologize $\mathcal{B}$ by using $\{[T]: T \in \mathcal{T}\}$ as a subbase. Each set $[T]$ is clopen in this topology and, because each branch is countable, the branch space is first countable. In addition the branch space is $T_{1}$. Later in the proof, we will need the following easily verified claim:

Claim 1: Suppose $\mathcal{C} \cup \mathcal{D}$ is a dense open subset of $\mathcal{B}$. Then $\mathcal{H}=\{S \in \mathcal{T}:[S] \subset \mathcal{C}$ or $[S] \subset \mathcal{D}\}$ is a dense open subset of $(\mathcal{T}, \Phi)$.

Claim 2: There is a set $\left\{\left(A_{\alpha}, D_{\alpha}, T_{\alpha}\right): \alpha<\underline{c}\right\}$ such that

(a) $A_{\alpha} \subset D_{\alpha} \subset Q$ and $T_{\alpha} \in \mathcal{T}$ for each $\alpha<\underline{c}$;

(b) if $\alpha \neq \beta$, then $T_{\alpha} \neq T_{\beta}$;

(c) whenever $\beta<\underline{c}$ and $A \subset D \subset Q$ and $T \in \mathcal{T}$, there is an $\alpha$ with $\beta<\alpha<\underline{c}$ such that $A_{\alpha}=A, D_{\alpha}=D$ and $T \prec T_{\alpha}$.

To show that such an indexing can be found, let $\mathcal{C}=\left\{\left(A, D, T^{\prime}\right): A \subset D \subset\right.$ $\left.Q, T^{\prime} \in \mathcal{T}\right\}$ and let $\mathcal{C}=\left\{\left(A_{\alpha}, D_{\alpha}, T_{\alpha}^{\prime}\right): \alpha<\underline{c}\right\}$ be any indexing of $\mathcal{C}$. Choose any $T_{0} \in \mathcal{T}$ with $T_{0}^{\prime} \prec T_{0}$ and for $\alpha>0$ replace $T_{\alpha}^{\prime}$ by some set $T_{\alpha}$ having $T_{\alpha}^{\prime} \prec T_{\alpha}$ and $T_{\alpha} \notin\left\{T_{\beta}: \beta<\alpha\right\}$. Notice that for fixed $A, D$, and $T$ with $A \subset D \subset Q$, there are $\underline{c}$-many triples of the form $\left(A, D, T^{\prime}\right)$ with $T \prec T^{\prime}$ so that, given $\beta, A, D$, and $T$ as in (c), there is an $\alpha>\beta$ with $A_{\alpha}=A, D_{\alpha}=D$, and $T \prec T_{\alpha}^{\prime}$. Replacing $T_{\alpha}^{\prime}$ by $T_{\alpha}$ as described produces the required $\left(A_{\alpha}, D_{\alpha}, T_{\alpha}\right)$.

Next we describe a recursion that produces the topology $\tau$ on $X=Q \times \mathcal{B}$. We will define a hierarchy $\left\{\tau_{\alpha}: \alpha<\underline{c}\right\}$ of topologies on $X$ as follows. Begin by letting $\tau_{0}$ be the usual product topology on $Q \times \mathcal{B}$. Next, if $0<\alpha<\underline{c}$ and we have defined $\tau_{\beta}$ for each $\beta<\alpha$, we consider two cases. In case $\alpha$ is a limit ordinal, let $\tau_{\alpha}$ be the topology for which $\bigcup\left\{\tau_{\beta}: \beta<\alpha\right\}$ is a base. In case $\alpha=\gamma+1$, consider the triple $\left(A_{\gamma}, D_{\gamma}, T_{\gamma}\right)$ and let $\tau_{\alpha}=\tau_{\gamma}$ unless each of the following holds:

(i) $D_{\gamma} \times\left[T_{\gamma}\right]$ is clopen in $\tau_{\gamma}$;

(ii) $\operatorname{Int}_{\tau_{\gamma}}\left(A_{\gamma} \times\left[T_{\gamma}\right]\right)=\emptyset$; and

(iii) $\operatorname{Int}_{\tau_{\gamma}}\left(\left(D_{\gamma}-A_{\gamma}\right) \times\left[T_{\gamma}\right]\right)=\emptyset$

and in that case, let $\tau_{\alpha}$ be the topology having $\tau_{\gamma} \cup\left\{A_{\gamma} \times\left[T_{\gamma}\right],\left(D_{\gamma}-A_{\gamma}\right) \times\left[T_{\gamma}\right]\right\}$ as a subbase. This recursion defines $\tau_{\alpha}$ for each $\alpha<\underline{c}$ and we let $\tau$ be the topology having $\bigcup\left\{\tau_{\alpha}: \alpha<\underline{c}\right\}$ as a base.

Claim 3: The topology $\tau$ has a subbase consisting of sets of the form $C \times[T]$ where one of the following holds:

(i) $C$ is clopen in the usual topology of $Q$;

(ii) for some $\gamma<\underline{c}, C \times[T]=A_{\gamma} \times\left[T_{\gamma}\right]$;

(iii) for some $\gamma<\underline{c}, C \times[T]=\left(D_{\gamma}-A_{\gamma}\right) \times\left[T_{\gamma}\right]$.

Consequently, $X$ is first countable, because each $(q, b) \in X$ belongs to only countably many of the above subbasic open sets.

Claim 4: The topology $\tau$ has a base consisting of clopen sets of the form $D \times[T]$. One can verify this assertion by an inductive proof that each $\tau_{\alpha}$ has a base of such sets, each being $\tau_{\alpha}$-clopen. Hence the space $(X, \tau)$ is completely regular. Further, it is a $\mathrm{T}_{1}$ space because $\tau_{0}$ is a $\mathrm{T}_{1}$-topology on $X$ and $\tau_{0} \subset \tau$. 
Claim 5: $(X, \tau)$ is paracompact.

To verify Claim 5, let $\mathcal{U}$ be an open cover of $X$. For $q \in Q$, let $\mathcal{T}(q)$ be the set of all $T \in \mathcal{T}$ such that $D_{T} \times[T] \subset U$ for some $U \in \mathcal{U}$ and for some clopen set $D_{T} \times[T]$ with $q \in D_{T}$. Let $\mathcal{M}(q)$ be the mimimal members (in the tree order) of $\mathcal{T}(q)$. Let $\mathcal{V}(q)=\left\{D_{T} \times[T]: T \in \mathcal{M}(q)\right\}$. Since clopen sets of the form $D \times[T]$ form a base, it is easy to check that $\{[T]: T \in \mathcal{M}(q)\}$ covers the branch space $\mathcal{B}$. By minimality, $\mathcal{M}(q)$ is an antichain in $\mathcal{T}$, so it follows that $\mathcal{V}(q)$ is a disjoint open partial refinement of $\mathcal{U}$ which covers $\{q\} \times \mathcal{B}$. Now for any point $\left(q^{\prime}, b\right) \in X, b$ is in a unique member $T$ of $\mathcal{M}(q)$, whence $Q \times[T]$ is an open neighborhood of $\left(q^{\prime}, b\right)$ that meets only one member of $\mathcal{V}(q)$. Thus $\mathcal{V}(q)$ is discrete, and so $\mathcal{U}$ has a $\sigma$-discrete open refinement. Hence $(X, \tau)$ is paracompact.

Claim 6: If $\mathcal{U}$ is a non-empty open subset of $(X, \tau)$ and if $\pi: X \rightarrow Q$ is first coordinate projection, then $\pi[\mathcal{U}]$ is infinite. Consequently, each set $\{q\} \times \mathcal{B}$ is closed and nowhere dense in $X$. Thus $X$ is not a Baire space because $X=\bigcup\{\{q\} \times \mathcal{B}$ : $q \in Q\}$.

To verify Claim 6, suppose there are a first ordinal $\alpha$ and a non-empty $U \in \tau_{\alpha}$ with $\pi[U]$ finite. Then $\alpha>0$ and $\alpha$ is not a limit ordinal, so $\alpha=\gamma+1$ for some $\gamma$. Note that $\tau_{\alpha} \neq \tau_{\gamma}$. Hence the three conditions of the inductive construction of $\tau_{\alpha}$ are satisfied and sets of the form $V, V \cap\left(A_{\gamma} \times\left[T_{\gamma}\right]\right)$ and $V \cap\left(\left(D_{\gamma}-A_{\gamma}\right) \times\left[T_{\gamma}\right]\right)$, where $V \in \tau_{\gamma}$, form a base for $\tau_{\alpha}$. Minimality of $\alpha$ yields that $U \notin \tau_{\gamma}$. Consider the case where $U=V \cap\left(A_{\gamma} \times\left[T_{\gamma}\right]\right)$, the other case being analogous. Because of condition (i) in Claim 2, we know that $D_{\gamma} \times\left[T_{\gamma}\right] \in \tau_{\gamma}$ so that $V \cap\left(D_{\gamma} \times\left[T_{\gamma}\right]\right) \in \tau_{\gamma}$. Minimality of $\alpha$ yields that $\pi\left[V \cap\left(D_{\gamma} \times\left[T_{\gamma}\right]\right)\right]$ is infinite. Because $\pi[U]$ is finite, the set $\pi[U] \times \mathcal{B}$ is closed in $\left(X, \tau_{\gamma}\right)$ so that the set $W=V \cap\left(D_{\gamma} \times\left[T_{\gamma}\right]\right)-(\pi[U] \times \mathcal{B})$ belongs to $\tau_{\gamma}$ and is non-empty. But $W \subset\left(D_{\gamma}-A_{\gamma}\right) \times\left[T_{\gamma}\right]$ so that $\operatorname{Int}_{\tau_{\gamma}}\left(\left(D_{\gamma}-A_{\gamma}\right) \times\left[T_{\gamma}\right]\right) \neq \emptyset$, contradicting part (iii) in the inductive construction of $\tau_{\alpha}$. Thus, Claim 6 holds.

Claim 7: If $\mathcal{G}_{0}$ and $\mathcal{G}_{1}$ are dense open subsets of $(X, \tau)$, then $\mathcal{G}_{0} \cap \mathcal{G}_{1}$ is dense in $X$. Hence $X$ is a Volterra space.

To verify Claim 7 , suppose (for contradiction) that some non-empty $\mathcal{U} \in \tau$ has $\mathcal{U} \cap \mathcal{G}_{0} \cap \mathcal{G}_{1}=\emptyset$. We may assume that $\mathcal{U}=D \times[T]$ (see Claim 4).

For $q \in Q$ and $e \in\{0,1\}$, let $\mathcal{G}(q, e)=\left\{b \in \mathcal{B}:(q, b) \in \mathcal{G}_{e}\right\}$. Then $\mathcal{G}(q, e)$ is a $G_{\delta^{-}}$ subset of the branch space $\mathcal{B}$. Find open sets $\mathcal{V}(q, e, n)$ in $\mathcal{B}$ such that $\mathcal{V}(q, e, n+1) \subset$ $\mathcal{V}(q, e, n)$ and $\mathcal{G}(q, e)=\bigcap\{\mathcal{V}(q, e, n): n \geq 1\}$. Let $\mathcal{O}_{q}=\mathcal{B}-\operatorname{cl}_{\mathcal{B}}(\mathcal{G}(q, 0) \cup \mathcal{G}(q, 1))$. Then for each $q \in Q$ and $n \geq 1$, the set $\mathcal{O}_{q} \cup \mathcal{V}(q, 0, n) \cup \mathcal{V}(q, 1, n)$ is a dense open subset of $\mathcal{B}$. According to Claim 1, each set $\mathcal{H}(q, n)=\left\{S \in \mathcal{T}:[S] \subset \mathcal{O}_{q}\right.$ or $[S] \subset$ $\mathcal{V}(q, 0, n)$ or $[S] \subset \mathcal{V}(q, 1, n)\}$ is dense and open in the auxiliary space $(\mathcal{T}, \Phi)$. Hence so is $\mathcal{H}=\bigcap\{\mathcal{H}(q, n): q \in Q, n \geq 1\}$.

Recall that $\mathcal{U}=D \times[T]$. Density allows us to choose $S \in \mathcal{H}$ with $T \prec S$. Then $[S] \subset \mathcal{O}_{q}$ or else for each $n \geq 1,[S] \subset \mathcal{V}(q, e, n)$ for some $e \in\{0,1\}$. Consider the case where $[S] \subset \mathcal{V}\left(q, 1, n_{i}\right)$ for infinitely many $n_{i}<n_{i+1}$, the other case being analogous. Then $[S] \subset \mathcal{G}(q, 1)$. Thus, either $[S] \subset \mathcal{O}_{q}$ or $[S] \subset \mathcal{G}(q, 0)$ or $[S] \subset \mathcal{G}(q, 1)$.

With $D$ as above, let $A=\{q \in D:[S] \subset \mathcal{G}(q, 0)\}$. It is easy to see that $A \times[S] \subset \mathcal{G}_{0}$ and $(A \times[S]) \cap \mathcal{G}_{1}=\emptyset$. We assert that $((D-A) \times[S]) \cap \mathcal{G}_{0}=\emptyset$. To verify that assertion, suppose

$$
\exists(q, b) \in((D-A) \times[S]) \cap \mathcal{G}_{0} .
$$

Because $q \in D-A$ we know that $[S] \not \subset \mathcal{G}(q, 0)$. Hence $[S] \subset \mathcal{G}(q, 1)$ or $[S] \subset \mathcal{O}_{q}$. In case $[S] \subset \mathcal{G}(q, 1)$, we have $(q, b) \in \mathcal{G}_{1}$ so that $(q, b) \in \mathcal{G}_{0} \cap \mathcal{G}_{1}$. Hence $(q, b) \notin \mathcal{U}=$ 
$D \times[S]$ because $\mathcal{U} \cap \mathcal{G}_{0} \cap \mathcal{G}_{1}=\emptyset$. Because $q \in D-A \subset D$, we are forced to conclude that $b \notin[T]$. However, $b \in[S] \subset[T]$ because $T \prec S$, and that contradiction shows that $[S] \subset \mathcal{G}(q, 1)$ cannot occur. In the remaining case, $[S] \subset \mathcal{O}_{q}$, so that our definition of $\mathcal{O}_{q}$ yields $[S] \cap \mathcal{G}(q, 0)=\emptyset$. Hence $b \notin \mathcal{G}(q, 0)$, i.e., $(q, b) \notin \mathcal{G}_{0}$, contrary to assumption $(*)$. Therefore $((D-A) \times[S]) \cap \mathcal{G}_{0}=\emptyset$ as asserted.

Now apply the special properties of our indexing $\left\{\left(A_{\alpha}, D_{\alpha}, T_{\alpha}\right): \alpha<\underline{c}\right\}$ as in Claim 2 to find some $\gamma<\underline{c}$ so large that the triple $\left(A_{\gamma}, D_{\gamma}, T_{\gamma}\right)$ has:

(i) $D \times[T]$ is clopen in $\tau_{\gamma}$; and

(ii) $A_{\gamma}=A, D_{\gamma}=D$ and $S \prec T_{\gamma}$.

Then, at stage $\gamma+1$ of the construction of the topology $\tau$, either:

(iii) $\operatorname{Int}_{\tau_{\gamma}}\left(A_{\gamma} \times[T \gamma]\right) \neq \emptyset$; or

(iv) $\operatorname{Int}_{\tau_{\gamma}}\left(\left(D_{\gamma}-A_{\gamma}\right) \times[T \gamma]\right) \neq \emptyset$; or

(v) we added the set $A_{\gamma} \times\left[T_{\gamma}\right]$ to the topology $\tau_{\gamma+1}$.

In any of these cases, we have a non-empty $\tau$-open set that is disjoint from either $\mathcal{G}_{0}$ or $\mathcal{G}_{1}$ and that is impossible because both $\mathcal{G}_{0}$ and $\mathcal{G}_{1}$ are dense in $(X, \tau)$. Thus, Claim 7 is established.

\section{Application to special First CAtegory Sets}

A subspace $X$ of a space $Y$ is said to be perfectly meager in $Y$ if $X \cap K$ is a firstcategory subset of $K$ whenever $K$ is a closed dense-in-itself subset of $Y$. A space $X$ is always of first category if every dense-in-itself subset $A$ of $X$ is first category in itself. It is well-known that for subsets $X$ of a complete separable metric space $Y$, $X$ is perfectly meager in $Y$ if and only if $X$ is always of first category $(\mathbb{K}$, Theorem 1 , page 516]).

Techniques from Section 2 can give a self-contained proof of a characterization of perfectly meager subsets of the unit interval $I=[0,1]$ that first appeared in [BHL].

4.1 Theorem. The following properties of a space $X$ are equivalent:

(a) $X$ is homeomorphic to a perfectly meager subset of $I$;

(b) $X$ is homeomorphic to a subspace of $I$ and whenever $A \subset X$ there is a countable set $B \subset A$ such that $B$ is dense in $A$ and $B$ is a $G_{\delta}$-subset of $X$;

(c) $X$ is a zero-dimensional separable metric space and whenever $A \subset X$ there is a countable set $B \subset A$ such that $B$ is dense in $A$ and $B$ is a $G_{\delta}$-subset of $X$.

Proof. Because assertions (b) and (c) are clearly equivalent, it is enough to show the equivalence of (a) and (b).

Suppose (a) holds and suppose $A \subset X$ is given. First consider the case where $A$ is dense in itself. Fix a countable dense subset $\left\{a_{n}: n \geq 1\right\}$ of $A$. Let $Y=\operatorname{cl}_{X}(A)$ and let $K=\operatorname{cl}_{I}(Y)$. Because $X$ is perfectly meager, so is $Y$, and $K$ is a closed, densein-itself subset of $I$. Hence $Y \cap K$ is first category in $K$ so there are relatively closed subsets $C_{n}$ of the space $K$ with $Y=Y \cap K \subset \bigcup\left\{C_{n}: n \geq 1\right\}$ and $\operatorname{Int}_{K}\left(C_{n}\right)=\emptyset$.

Consider the relatively open subset $G_{n}=Y-C_{n}$ of $Y$. We claim that $G_{n}$ is dense in $Y$. If not, then there exist a point $p \in Y$ and an interval $(a, b) \subset I$ such that $p \in(a, b) \cap Y \subset C_{n}$. Because $Y$ is dense in $K$, we have $p \in(a, b) \cap K \subset$ $\operatorname{cl}_{K}((a, b) \cap Y) \subset \operatorname{cl}_{K}\left(C_{n}\right)=C_{n}$ showing that $p \in \operatorname{Int}_{K}\left(C_{n}\right)$ which is impossible. Thus, $G_{n}$ is a dense relatively open subset of $Y$.

Because $A$ is also dense in $Y$, the set $A \cap G_{n}$ is dense in $Y$. Thus, for each $a_{n}$ in the countable dense set chosen above, we can find a sequence $\{a(n, j): j \geq 1\}$ of points of $A \cap G_{n}$ that converges to $a_{n}$. Then the set $H_{n}=\{a(n, j): j \geq 1\}$ is a $G_{\delta^{-}}$subset of $Y, H_{n} \subset G_{n}$, and $H_{n} \subset A$. Because $\bigcap\left\{G_{n}: n \geq 1\right\}=\emptyset$, Lemma 2.3 
shows that the set $B=\bigcup\left\{H_{n}: n \geq 1\right\}$ is a $G_{\delta}$-subset of the space $Y$. But, because $Y$ is closed in $X, Y$ is a $G_{\delta}$-subset of $X$ so that $B$ is also a $G_{\delta}$-subset of $X$. Finally, note that each point $a_{n}$ is a limit point of $B$, so that $B$ is dense in $A$.

Now consider the general case, where the subset $A$ of $X$ might not be dense-initself. Let $A_{0}=\{a \in A: a$ is an isolated point of the set $A\}$. Then $A_{0}$ is countable and is a $G_{\delta}$ in $X$. Let $A_{1}=A-\operatorname{cl}_{X}\left(A_{0}\right)$. Then $A_{1}$ is dense in itself so that the first part of our argument yields a countable set $B_{1} \subset A_{1}$ that is dense in $A_{1}$ and is a $G_{\delta}$-subset of $X$. Letting $B=A_{0} \cup B_{1}$ we obtain the desired set $B$. Thus (a) implies (b).

To prove that (b) implies (a), suppose that $X$ satisfies (b) of the theorem, and suppose $K$ is a dense-in-itslf subset of $I$. We must show that the set $A=K \cap X$ is first category in $K$. Find the countable set $B \subset A$ as described in (b), and write $B=\bigcap\left\{X \cap V_{n}: n \geq 1\right\}$ where each $V_{n}$ is open in $I$. It is easy to see that $A \subset B \cup \bigcup\left\{X-V_{n}: n \geq 1\right\}$ so that, with $C=\mathrm{cl}_{K}(A)$, we have $A \subset$ $B \cup \bigcup\left\{C-V_{n}: n \geq 1\right\}$. Because $B \subset V_{n}$ and $B$ is dense in $A$, which is dense in $C$,we see that $V_{n}$ is dense in $C$. Hence $C-V_{n}$ is a closed, nowhere dense subset of $C$. Therefore each $C-V_{n}$ is also a closed nowhere dense subset of $K$. Because $K$ is dense-in-itself, the set $\{b\}$ is closed and nowhere dense in $K$ for each $b \in B$. Thus $A \subset B \cup \bigcup\left\{C-V_{n}: n \geq 1\right\}$ shows that $A=K \cap X$ is first category in $K$ as required.

It is natural to ask whether the hypothesis of zero-dimensionality can be dropped from $(4.1(\mathrm{c}))$. The answer is "No" as can be seen from the fact that there exist $\lambda$-sets (i.e., separable metric spaces every countable subset of which is a relative $\left.G_{\delta}\right)$ of every possible dimension $(\underline{\mathrm{K}}$, Theorem 5, p. 520]) and any $\lambda$-set clearly satisfies all of the conditions of 4.1(c) except for 0-dimensionality. However, the remaining conditions in 4.1(c) turn out to describe exactly the class of separable metric spaces that are always of first category. In fact, from the results in Section 2 we obtain the more general characterization of metric spaces that are always of first category, as follows.

4.3 Proposition. For any metric space $X$, the following are equivalent:

(a) $X$ is always of first category;

(b) For every subset $A \subseteq X$, there is a $\sigma$-closed discrete subset $B \subset A$ that is dense in $A$ and is a $G_{\delta}$-subset of $X$.

Proof. It is clear that (b) implies (a). To prove the converse, suppose $X$ satisfies (a) and $A \subset X$. Let $B_{0}$ be the set of isolated points of $A$ and let $A_{1}=A-\operatorname{cl}_{X}\left(B_{0}\right)$. Then $B_{0}$ is $\sigma$-closed discrete in $X$. Further, $A_{1}$ is dense in itself, whence so is $\operatorname{cl}_{X}\left(A_{1}\right)$, so that by $(\mathrm{a}), \mathrm{cl}_{X}\left(A_{1}\right)$ is of first category in itself. Now apply Corollary 2.5 to find a dense subset $B_{1}$ of $A_{1}$ that is a $\sigma$-closed discrete $G_{\delta}$-subset of $\operatorname{cl}_{X}\left(A_{1}\right)$. Then $B_{1}$ is also a $\sigma$-closed discrete $G_{\delta}$-subset of $X$. Now let $B=B_{0} \cup B_{1}$.

4.4 Remark. In case $X$ is separable metric, then the subset $B$ in $4.3(\mathrm{~b})$ will be countable.

\section{REFERENCES}

[AN] Arhangel'skii, A. and Nedev, S., Some remarks on semi- metrizable spaces and their subspaces, Comptes Rendu Acad. Bulgare Sci., 31(1978), 499-500. MR 80c:54026]

[BHL] Bennett, H., Hosobuchi, M., and Lutzer, D., On weakly perfect generalized ordered spaces, to appear. 
[vD] van Douwen, E. K., Applications of maximal topologies, Topology and its Applications, 51 (1993), 125-139. MR 94h:54012

[E] Engelking, R., General Topology, Heldermann Verlag, Berlin, 1989. MR 91c:54001

[G1] Gruenhage, G., Generalized metric spaces, pp. 425-501, in Handbook of Set Theoretic Topology, ed. by Kunen, K., and Vaughan, J., North Holland, 1984, Amsterdam. MR 86h:54038

[G2] Gruenhage, G., Irreducible restrictions of closed mappings, 8th Prague Topological Symposium on General Topology and Its Relations to Modern Analysis and Algebra (1996). Topology Appl. 85 (1998), no. 1-3, 127-135. CMP 98:11

[GGP] Gauld, D., Greenwood, D., and Piotrowski, Z., On Volterra spaces II, Annals of the New York Academy of Sciences, 806 (1996), 169-173. MR 97m:54052

[GP] Gauld, D., and Piotrowski, Z., On Volterra spaces, Far East J. Math. Sci. 1 (1993), $209-214$. MR 94k:54070

[H] Hewitt, E. A problem of set-theoretic topology, Duke Math J. 10(1943), 309-333. MR 5:46e

[He] Heath, R.W., Screenability, pointwise paracompactness, and metrization of Moore spaces, Canad. J. Math 16 (1964), 763-770. MR 29:4033

[K] Kuratowski, K., Topology, Volume 1, Academic Press, 1966, New York. MR 36:840

[P] Pytkeev, E.G., Maximally resolvable spaces, Trudy Mat. Inst. Steklov, 154 (1983), $209-213$. MR 85c:54039

[RM] Reed, G.M. and McIntyre, D.W., A Moore space with caliber $\left(\omega_{1}, \omega\right)$ but without caliber $\omega_{1}$, Topology and its Applications, 44 (1992), 325-329. MR 93i:54019

[T] Todorcevic, S., Trees and linearly ordered sets, pp. 235-293, in Handbook of Set Theoretic Topology, ed. by Kunen, K., and Vaughan, J., North Holland, 1984, Amsterdam. MR 86h:54040

[V] Volterra, V., Alcune osservasioni sulle funzioni punteggiate discontinue, Giornale di Matematiche 19 (1881), 76-86.

Department of Mathematics, Auburn University, Auburn, Alabama 36849-5310

E-mail address: garyg@mail.auburn.edu

Department of Mathematics, College of William \& Mary, Williamsburg, Virginia 23187

E-mail address: lutzer@math.wm.edu 